\title{
Le collectif face au big data : interprétation partagée et retro-validation des données LiDAR du site d'El Infiernillo, Michoacán, Mexique
}

\author{
The collective faced with big data: shared interpretation and retro- \\ validation of LiDAR data from the El Infiernillo site, Michoacán, Mexico
}

\author{
Marion Forest ${ }^{1}$, Laurent Costa $^{2}$, Grégory Pereira ${ }^{3}$ \\ ${ }^{1}$ Postdoctoral fellow, Anthropology Department, Brigham Young University, marion_forest@byu.edu \\ ${ }^{2}$ Ingénieur de recherche CNRS, UMR 7041 ArScAn - Archéologies et Sciences de l'Antiquité, \\ laurent.costa-asi@mae.u-paris10.fr \\ ${ }^{3}$ Directeur de recherche, CNRS, UMR 8096, Archéologie des Amériques, gregory.pereira@mae.u-paris10.fr
}

RÉSUMÉ. L'obtention en 2015 d'une couverture LiDAR (Light Detection and RAnging) de l'intégralité du complexe géologique du Malpaís de Zacapu, dans le Centre-Ouest Mexicain, a radicalement transformé les modalités d'étude de ce secteur de Mésoamérique. A l'image des expériences similaires menées dans le Bassin de Pátzcuaro ou dans la région maya, l'acquisition de données LiDAR confronte l'archéologue à de nombreuses reconfigurations méthodologiques et paradigmatiques : la chaîne opératoire de son travail, en particulier de son travail de terrain peut être modifiée. Par ailleurs, la masse de données à stocker, gérer, analyser, est radicalement multipliée. Des dispositifs et stratégies de traitement adaptés doivent, face à cela, être conceptualisés et développés. Enfin, des tests approfondis et des rétroanalyses doivent être menés pour valider non seulement l'utilisation de la donnée LiDAR, mais aussi les outils de recherche construits dans ce cadre.

En partant de données acquises et d'interprétations réalisées au préalable dans le cadre d'opérations de terrain "classiques" et en nous basant sur un cas d'étude, le site archéologique d'El Infiernillo, nous discutons des potentialités des plateformes de traitement cartographiques collaboratives et de la gestion des données " télé-interprétées ».

ABSTRACT. The acquisition, in 2015, of a high-resolution LiDAR (Light Detection and RAnging) elevation model for the entire geological complex of the Malpaís of Zacapu, West Mexico, has deeply influenced the research methods and general approach to this region of Mesoamerica in recent years. Similar experiments have been conducted in the Pátzcuaro Basin or in the Mayan area, where LiDAR data have also left archaeologists facing methodological and paradigmatic changes: the modus operandi of archaeological work, particularly the field component, has been transformed. Besides the difference in the general research process, the large volume of data available forms a challenge in terms of storage, data management and analysis processes. Specific and adapted strategies and tools thus have to be designed and developed. Finally, tests and retro-analyses have to be run to validate the use of LiDAR data and associated tools in archaeological research.

In this article, we focus on two topics, using the case study of the archaeological site of El Infiernillo: first, we present and discuss the potential of methods based on collective "big" data processing (in particular webGIS); and second we test the validity of remotely mapped information by comparing it to data obtained using traditional ground survey methods.

MOTS-CLÉS. LIDAR, archéologie, cartographie en ligne, système d'information géographique, urbanisme, Mésoamérique.

KEYWORDS. LiDAR, archaeology, webmapping, Geographic Information System, urbanism, Mesoamerica.

\section{Introduction}

Les milieux forestiers constituent des environnements particulièrement favorables à la préservation des vestiges archéologiques. Faiblement impactés par les activités anthropiques contemporaines, ces espaces ont fréquemment permis la préservation de véritables paysages fossiles perceptibles à travers les variations topographiques du terrain. Si le couvert végétal a longtemps constitué un obstacle à l'appréhension globale et à la cartographie de ces vestiges, l'introduction, au cours des dernières années, de la technologie LIDAR constitue une avancée considérable que certains auteurs n'ont pas hésité à qualifier de révolution pour la recherche archéologique (Chase et al. 2012). La Mésoamérique (C) 2018 ISTE OpenScience - Published by ISTE Ltd. London, UK - openscience.fr 
offrant de nombreux espaces propices à ce type d'approche, il n'est pas surprenant que la télédétection LIDAR y jouisse d'un succès certain (voir par exemple Fernandez-Diaz et al. 2014, Fisher et Leisz 2013, Hutson 2015, Golden et al. 2016, Von Schwerin et al. 2016). Les espaces boisés qui parsèment ce vaste territoire abritent en effet un grand nombre de sites archéologiques majeurs dont l'abandon a été souvent définitif, garantissant une bonne conservation des modifications de surface apportées par ces sociétés anciennes.

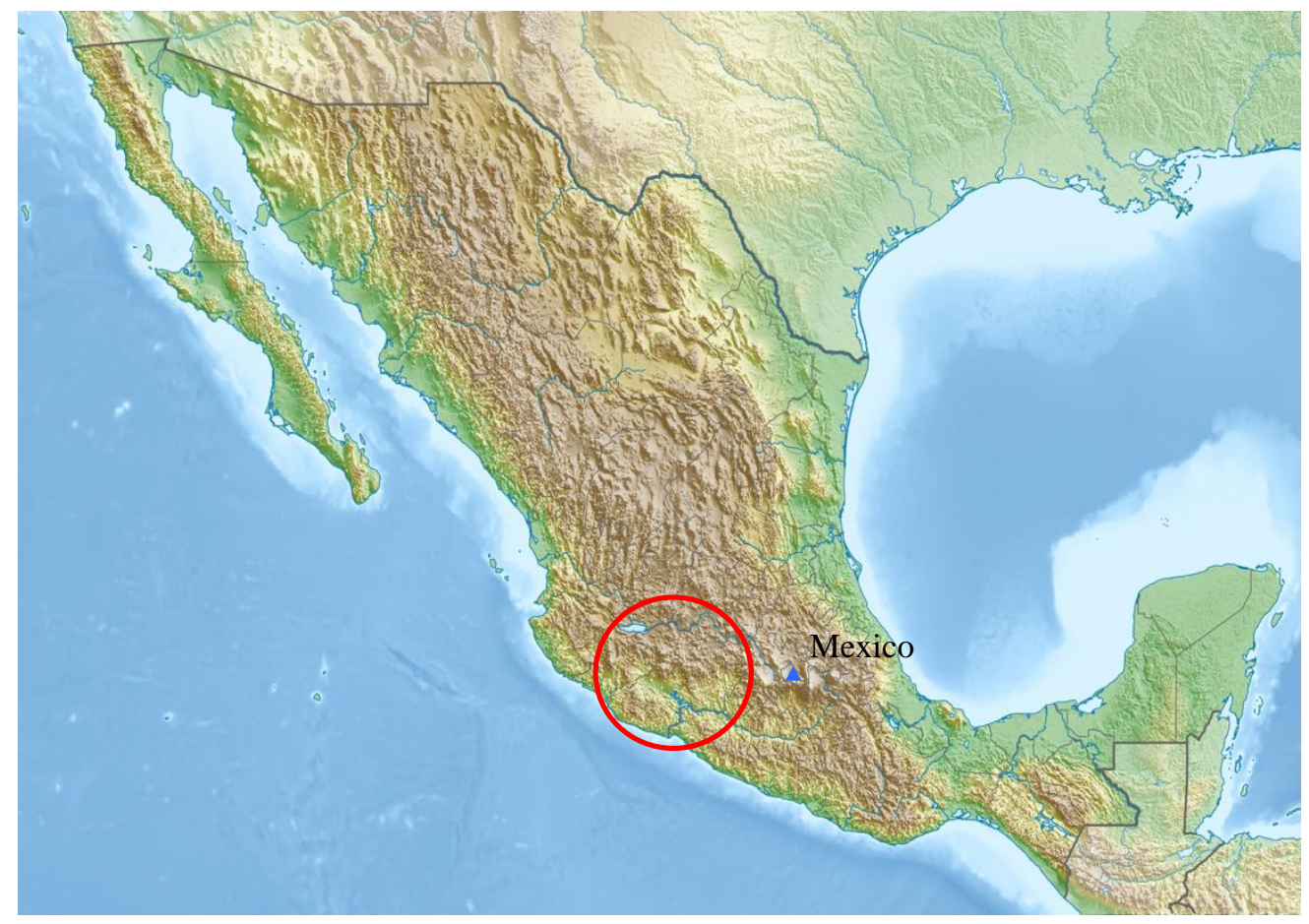

Figure 1. Carte de situation de la zone du Michoacán

Si les études dans les forêts tropicales humides des basses terres se sont multipliées, les hautes terres volcaniques du Michoacán (Mexique) fournissent également un certain nombre de contextes remarquables. Fortement modelés, tout au long du quaternaire, par une intense activité volcanique, les paysages de la région sont ponctués de nombreux malpaís, terme espagnol qui désigne les coulées de lave quaternaires qui se caractérisent par des surfaces rocheuses tourmentées généralement considérées par les populations modernes comme peu propices aux activités humaines. Or, plusieurs de ces formations ont fait l'objet d'une intense occupation précolombienne qui a inévitablement modifiée la morphologie naturelle des coulées (cf Michelet, 1998, Fisher et Leisz 2013). La plupart de ces sites ont été occupés au cours des cinq siècles qui précédèrent l'arrivée des Espagnols au début du XVI ${ }^{\text {ème }}$ siècle et nombre d'entre eux ont un lien étroit avec le phénomène d'émergence du royaume tarasque qui dominait la région à l'époque du contact. Les recherches conduites actuellement dans la région dans le cadre des projets Uacusecha ${ }^{1}$ et Mésomobile ${ }^{2} \mathrm{~s}^{\prime}$ intéressent plus particulièrement à un complexe de sites à caractère urbain localisés sur une zone de malpaís situé au nord-ouest de la ville actuelle de Zacapu (Migeon 1990, Michelet 1998, Forest 2014). Si de nombreuses recherches avaient été conduites précédemment, la réalisation en 2015 d'une couverture LIDAR de $92 \mathrm{~km}^{2}$ opérée par le National Center for Airborn Laser Mapping (University of Houston) a permis l'acquisition d'un ensemble considérable de données qui permettent de renouveler largement les recherches sur ce secteur mais soulève également des questions importantes face au traitement de la masse des informations obtenues.

\footnotetext{
${ }^{1}$ Mission archéologique dirigée par Grégory Pereira et financé par la commission des fouilles du Ministère des Affaires Etrangères et du Développement International et l'UMR 8096 " Archéologie des Amériques ».

${ }^{2}$ Programme financé par l'ANR porté par Véronique Darras.

(C) 2018 ISTE OpenScience - Published by ISTE Ltd. London, UK - openscience.fr 
La présente contribution fait état d'une stratégie qui a été mise en place pour remédier à ce problème et qui propose, grâce à l'utilisation d'une plate-forme de webmapping dédiée, de procéder à une saisie collaborative des formes révélées par les images LIDAR dans le but de constituer une première cartographie d'un des principaux établissements de la zone : le site d'El Infiernillo. Cette opération, réalisé dans le cadre d'un exercice universitaire incluant un groupe d'étudiants du Master Environnement de l'Université Paris 1 Panthéon/Sorbonne, avait aussi une vocation pédagogique et un souci de formation par la recherche. Dans les lignes qui suivent, nous présentons les résultats obtenus après une présentation des antécédents des recherches dans la zone d'étude.

\section{L'urbanisme des agglomérations du Malpaís : Antécédents et support d'analyse des données LiDAR}

\section{Bref historique des recherches}

L'exploration des agglomérations archéologiques du Malpaís de Zacapu débute dès la fin du XIX ${ }^{\mathrm{e}}$ siècle, mais ce n'est qu'au début des années 1980 que les travaux menés prennent une dimension scientifique. Des prospections systématiques et plusieurs campagnes de sondages, sont alors conduites, dans le cadre du Projet Michoacán I, par des équipes françaises et mexicaines, dans l'ancien bassin palustre de Zacapu. Cette première phase de travaux avait pour ambition d'établir la carte archéologique inédite de la région et de dater les établissements repérés. Les sites construits sur le Malpaís avaient bien sûr, par leur importante taille et leur architecture à caractère monumental, déjà retenus l'attention des explorateurs et archéologues de passage (Lumholtz 1986 [1904], Caso 1930, Freddolino 1973). Mais pour la première fois, une évaluation plus claire des vestiges était faite, avec notamment, une estimation de l'extension et des composantes des cités. Le site de Las Milpillas (Mich. 95) a été, dans le cadre de travaux plus poussés, presque entièrement topographié, tandis que quelques hectares du site de Malpaís Prieto (Mich. 31) avaient été levés (Michelet et al. 1988, Michelet 1992 : 40, Migeon 1990). Puis entre 1994 et 1996, une nouvelle série de travaux, menée dans le cadre du Projet Michoacán III (Michelet 1998), a permis à cette évaluation globale de s'affiner. Les sites d'El Infiernillo et Malpaís Prieto ont alors fait l'objet d'un inventaire et d'une carte de distribution des vestiges bâtis (Figure 2).

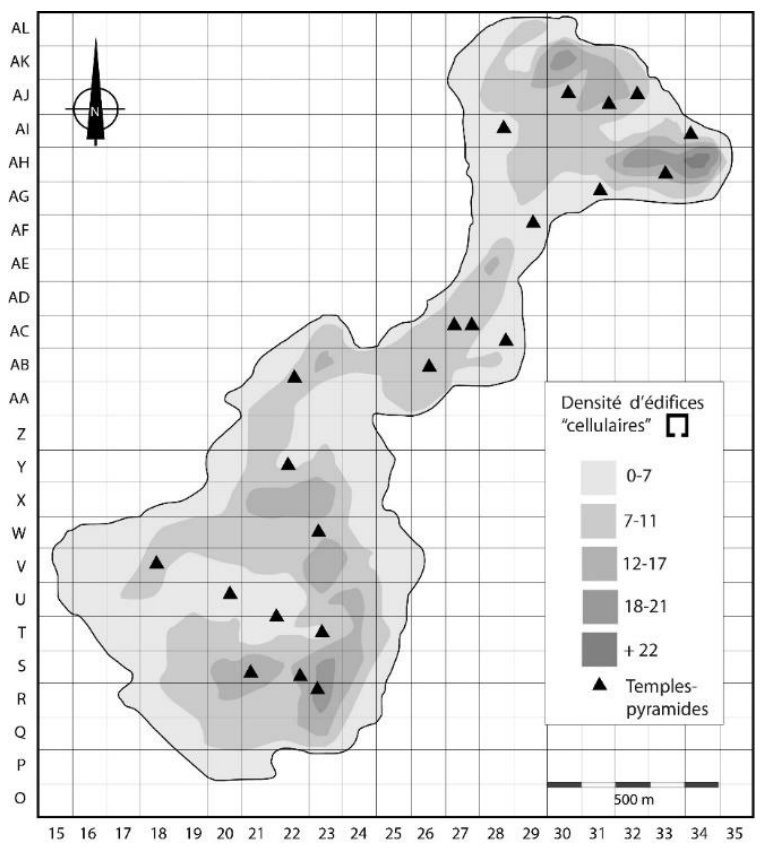

Figure 2. Carte de densité du site d'El Infiernillo, réalisée en 1994 après la prospection systématique du site (d'après Michelet 1992) 
Entre 1996 et 2008, aucune opération de terrain ne fut menée sur les agglomérations du Malpaís, l'intérêt des spécialistes s'étant déplacé aux régions voisines, peu explorées. C'est finalement en 2008 qu'une nouvelle campagne de terrain fut organisée, pour documenter le site de Malpaís Prieto (Forest 2008). S'en suivirent deux autres années de relevés détaillés et géo-référencés de ce site, puis des sites voisins d'El Palacio (partiel), El Infiernillo (partiel) et une campagne de vérification et géoréférencement du plan topographique du site de Las Milpillas (Forest 2014). A l'issue de ces terrains (Tableau 1), les quatre cités du Malpaís disposaient d'un relevé, complet ou partiel permettant une analyse avancée et comparative des quatre cités.

\begin{tabular}{|c|c|c|c|c|}
\hline Opération & Références & Technique de relevé & Données/Couverture & Limites \\
\hline \multirow[b]{2}{*}{$1983-1985$} & \multirow{2}{*}{$\begin{array}{c}\text { Projet Michoacán I } \\
\text { (Michelet et al. } \\
\text { 1988, Michelet } \\
\text { 1992, Migeon } \\
\text { 1990) }\end{array}$} & $\begin{array}{l}\text { Localisation sur carte } \\
\text { topo. } 1 / 50000\end{array}$ & $\begin{array}{c}\text { Géo-localisation des sites } \\
\text { archéologiques }\end{array}$ & $\begin{array}{l}\text { Pas de } \\
\text { données } \\
\text { surface }\end{array}$ \\
\hline & & $\begin{array}{c}\text { Relevés } \\
\text { topographique à } \\
\text { l'alidade et au } \\
\text { théodolite }\end{array}$ & $\begin{array}{c}70 \% \text { du site de Las } \\
\text { Milpillas, } 13 \% \text { du site de } \\
\text { Malpaís Prieto }\end{array}$ & $\begin{array}{c}\text { relevé } \\
\text { partiels non } \\
\text { géoréférencés }\end{array}$ \\
\hline 1994-1996 & $\begin{array}{l}\text { Projet Michoacán } \\
\text { III } \\
\text { (Michelet 1998) }\end{array}$ & $\begin{array}{l}\text { Comptage par unités } \\
\text { de } 1 \text { hectare, } \\
\text { positionnement des } \\
\text { soubassements } \\
\text { pyramidaux }\end{array}$ & $\begin{array}{c}\text { 100\% d'El Infiernillo et El } \\
\text { Malpaís Prieto }\end{array}$ & $\begin{array}{c}\text { Localisation } \\
\text { générale/pas } \\
\text { de relevé } \\
\text { systématique } \\
\text { du bâti }\end{array}$ \\
\hline $2008-2010$ & $\begin{array}{l}\text { Projet Uacúsecha } \\
\text { (Forest 2008, 2014) }\end{array}$ & Croquis GPS & $\begin{array}{c}\text { 100\% Malpaís Prieto ; } \\
\text { 60\% El Palacio ; 70\% Las } \\
\text { Milpillas ; 5\% El } \\
\text { Infiernillo } \\
\text { Analyse géostatistique }\end{array}$ & $\begin{array}{l}\text { Erreur du } \\
\quad \text { GPS } \\
\text { Couverture } \\
\text { incomplète }\end{array}$ \\
\hline $2010-2013$ & $\begin{array}{l}\text { Projet Uacúsecha } \\
\text { (Pereira et Forest } \\
\text { 2011, Pereira et al. } \\
\text { 2012) }\end{array}$ & $\begin{array}{c}\text { Relevés } \\
\text { tridimensionnels à la } \\
\text { station totale }\end{array}$ & $\begin{array}{l}\text { Relevé en 3D des zones de } \\
\text { fouilles de Malpaís Prieto }\end{array}$ & Partiel \\
\hline 2015 & $\begin{array}{c}\text { Projet Mésomobile } \\
\text { (Pereira et al. 2016, } \\
\text { Zimmer 2016) }\end{array}$ & $\begin{array}{l}\text { Couverture LiDAR } \\
\left(92 \mathrm{~km}^{2} \text {, env. } 10\right. \\
\text { points } / \mathrm{m}^{2}, \text { précision : } \\
1 \text { pixel = } 50 \mathrm{~cm})\end{array}$ & $\begin{array}{l}\text { Couverture complète du } \\
\text { Malpaís de Zacapu }\end{array}$ & $\begin{array}{l}\text { Interprétation } \\
\text { à distance }\end{array}$ \\
\hline
\end{tabular}

Tableau 1. Synthèse des travaux de relevés planimétriques et topographiques réalisés sur les quatre agglomérations urbaines du Malpaís de Zacapu depuis 1983.

\section{Interprétations}

Les relevés planimétriques et topographiques les plus complets ont été réalisés à Malpaís Prieto et Las Milpillas. Ces sites ont tous deux fait l'objet de fouilles extensives, permettant une interprétation fonctionnelle du bâti. Les plans des deux agglomérations, contemporaines, distantes de $3 \mathrm{~km}$ à vol d'oiseau, ont été analysés systématiquement. Parmi les caractéristiques organisationnelles fondamentales de ces cités, notons ici l'ultra-urbanisme (Forest 2014), la densité importante de constructions, l'homogénéité morphologique et la hiérarchisation sociale de l'espace. 
Les agglomérations du Malpaís ont été construites sur un terrain principalement, voire uniquement rocheux. Le bâti et une partie des dispositifs de circulation ont donc été réalisés en blocs d'andésite locale plus ou moins taillée. Les fouilles archéologiques ont documenté par ailleurs l'usage de matériaux périssables (terre et torchis) pour la partie supérieure des murs des habitations et des temples, et les greniers aériens destinés au stockage de denrées alimentaires par l'unité domestique. Malgré cela, les vestiges en pierre restent très bien conservés et le plan de toutes les composantes des cités est généralement très clairement identifiable. Tous les espaces utilisés par l'homme sont magistralement aménagés et les vestiges, bien conservés.

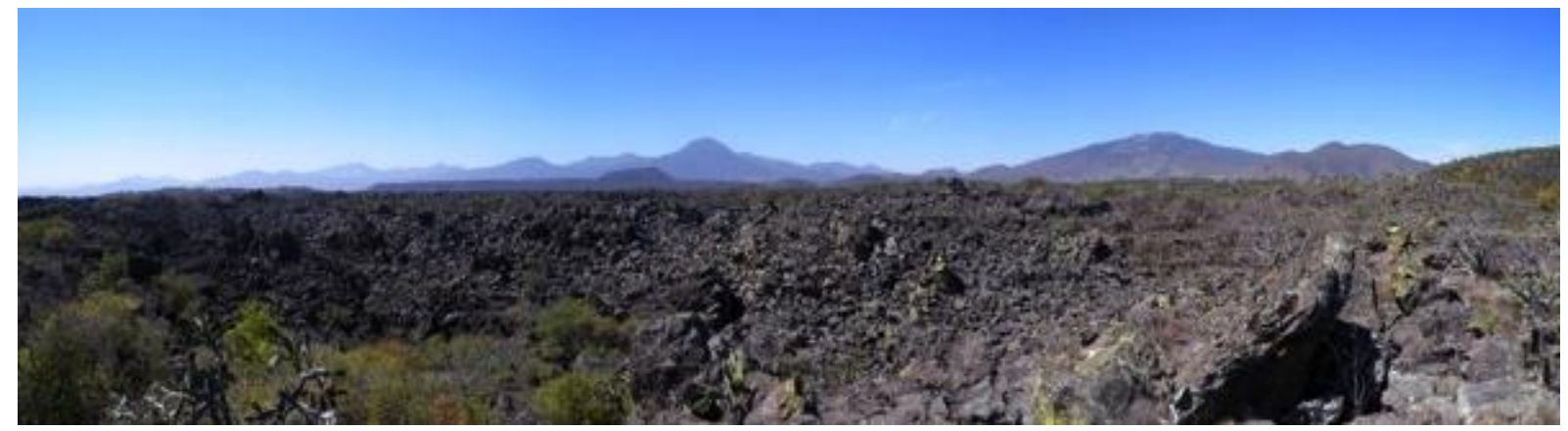

Figure 3. Vue du plateau du Malpaïs et son substrat de roches volcanique irrégulières.

Le site de Malpaís Prieto en tête, les agglomérations présentent une densité d'occupation et d'aménagement très haute. Cette densité correspond à la constitution d'un réseau d'habitat important, dans des espaces au coût urbanistique important (matériel et main d'œuvre) et à l'accessibilité complexe. Sur ces 37 hectares, le site de Malpaís Prieto compte presque 1200 parcelles terrassées, 1 000 structures d'habitation, auxquelles s'ajoutent les espaces religieux, le réseau de circulation. Une autre caractéristique est l'homogénéité morphologique et architecturale. L'usage d'un même matériau - la roche andésitique directement issue des coulées — dans chaque cité et dans l'ensemble du Malpaís, confère aux cités une grande similarité d'aspect. Mais c'est la tradition architecturale commune qu'il faut souligner : la typologie architecturale est très stable et homogène, et finalement assez réduite. Il est donc relativement aisé de placer une structure donnée dans l'une ou l'autre des catégories, distinctes les unes des autres. Ce phénomène d'homogénéité morphologique est notable, mais n'empêche pas l'existence de variantes locales interprétées comme le résultat de problématiques contextuelles et environnementales. Les caractéristiques topographiques, pédologiques, la couverture végétale et l'accès aux ressources extérieures possèdent, pour chacune des quatre cités, des spécificités, qui sans révision systématique sur le terrain, s'avèrent plus difficile à identifier et interpréter.

Le modèle d'organisation sociospatial des agglomérations du Malpaís est fondé sur plusieurs échelles structurelles : l'unité d'habitation (pouvant regrouper une ou plusieurs maisons, des espaces d'activité ouvert, des structures de stockage), le voisinage (fondé sur la mutualisation d'espaces d'activité domestique, de circulation et de stockage domestique), la zone résidentielle (qui structure, via la topographie et le réseau viaire, les espaces résidentiels aux espaces religieux distribués dans la cité) et le site (qui comprend l'ensemble, et présente une hiérarchisation générale polarisée par son épicentre civico-cérémoniel et sa zone de transit intra/extra-site). L'élite, représentée spatialement par un habitat de grandes dimensions, et des voisinages peu denses, est en connexion directe avec les lieux de pouvoirs, tandis que l'habitat commun, de surface petite à moyenne et de densité plus forte, occupe le reste des espaces disponibles, jusqu'aux limites de l'agglomération.

L'analyse comparative approfondie de Malpaís Prieto et Las Milpillas ne peut être étendue aux deux autres cités, même si les données recueillies sur ces dernières invitent à de nombreuses conclusions générales. Ensuite, certains tests géostatistiques ont été limités par la basse résolution du modèle d'élévation disponible (notamment les analyses d'inter-visibilités et de circulation). Enfin, la couverture obtenue restait très centrée sur les quatre agglomérations principales, sans prendre en compte les 
établissements plus petits pouvant exister en périphérie. L'exhaustivité de relevé pédestre restait une ambition impossible.

\section{L'apport des données LiDAR : résolution et exploitation collaborative de l'information}

L'obtention de la couverture LiDAR du Malpaís de Zacapu en 2015, grâce au programme de recherche Mésomobile («Mobilités et mutations politiques dans le Centre-ouest du Mexique" financé par l'ANR) a ouvert de nouvelles perspectives sur l'étude de ce complexe urbain sans précédent dans la région : une couverture intégrale du secteur avec une précision de relevé permettant non seulement l'obtention d'un modèle numérique d'élévation précis (valeur du pixel $=50 \mathrm{~cm}$ ), mais permettant aussi une prise en compte intégrale et continue des très nombreux vestiges archéologiques conservés pour l'ensemble de la zone. La vision des agglomérations du Malpaís et surtout l'organisation et la composition de leur périphérie directe ou plus éloignée, était dès lors transformée. Le potentiel pour la recherche archéologique était donc important, mais le traitement de ces milliers de données confrontait les chercheurs de l'équipe à des problématiques et des contraintes fortes en terme de gestion et manipulation de l'information. Le simple repérage des vestiges archéologiques sur les différentes versions du modèle numérique de terrain, constituait une tâche énorme. Par ailleurs, l'usage variable (problématique et méthodes) et compartimenté de la base de données LiDAR par les chercheurs (membres des projets Uacúsecha et Mésomobile), multipliait les supports et dispersait les analyses à l'échelle individuelle. Dans le cadre du programme Mésomobile, plusieurs outils de travail collaboratif ont donc été mis en œuvre afin de pallier les problèmes de management complexe de ces données massives.

Pour tenter de répondre à cette problématique, une plate-forme d'information géographique en ligne, plus généralement appelée webmapping ou webGIS a été développée. Il s'agissait de permettre aux différents intervenants d'accéder à une interface cartographique commune donnant accès en visualisation aux données LIDAR et en édition aux interprétations de ces données. Cela permettait à chaque participant de mener individuellement un travail de vectorisation des vestiges archéologiques reconnus sur l'image LiDAR, tout en les rendant visibles en temps réel par le collectif, via l'interface dématérialisée.

C'est la plateforme Reagit développée dans le cadre du programme ArchéoFab qui a été choisie (Costa, Desachy 2018). Centrée sur une base de donnée MYSQL elle permet au travers d'une interface html de proposer aux utilisateurs une interface personnalisée de navigation dans les données cartographiques et d'offrir des fonctionnalités de gestion et d'édition collaborative. L'utilisateur par le biais d'un simple navigateur (Firefox, chrome) peut visualiser les données LiDAR, interroger et enrichir les données précédemment saisies par le groupe de recherche. Deux couches de données dérivées des données LiDAR ont été intégrée à l'interface et rendues disponibles pour les chercheurs : l'ombrage (orientation...) et les pentes. Le choix de ces deux indicateurs uniques, parmi les plus classiques, s'explique pour deux raisons :

- Premièrement il s'agissait des deux indicateurs les plus lisibles parmi les diverses possibilités disponibles. La qualité du rendu permettait une interprétation assez aisée des structures archéologiques.

- Deuxièmement, pour faciliter la compréhension de l'interface et permettre au public cible, à savoir des étudiants non formés à ces technologies ou à ces interprétations, de s'approprier l'outil, nous avons préféré privilégier la simplicité de l'interface et des données. 


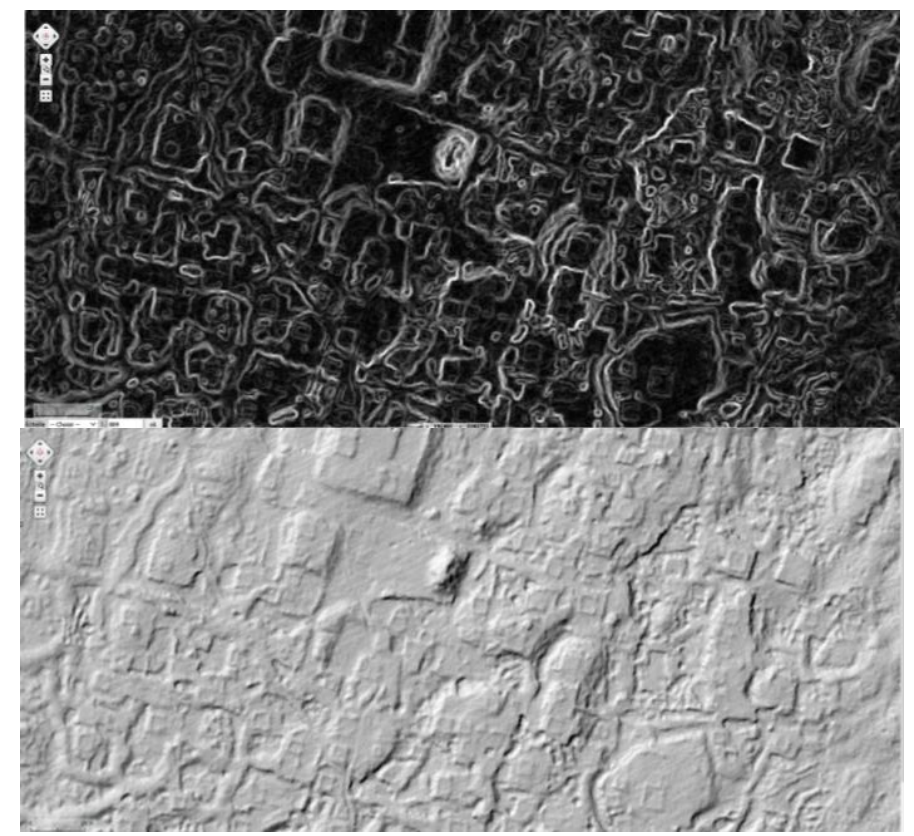

Figure 4. Deux indicateurs ont été mis à disposition : les classes de pente et les ombrages (nord-ouest) / Un compte permettant d'accéder à la plateforme de Web Mapping Recif-Reagit / Une portion d'espace affecté à un apprenant / un cahier des charges simple / un travail personnel de la part des apprenants / Discussion autour des résultats de l'interprétation collective

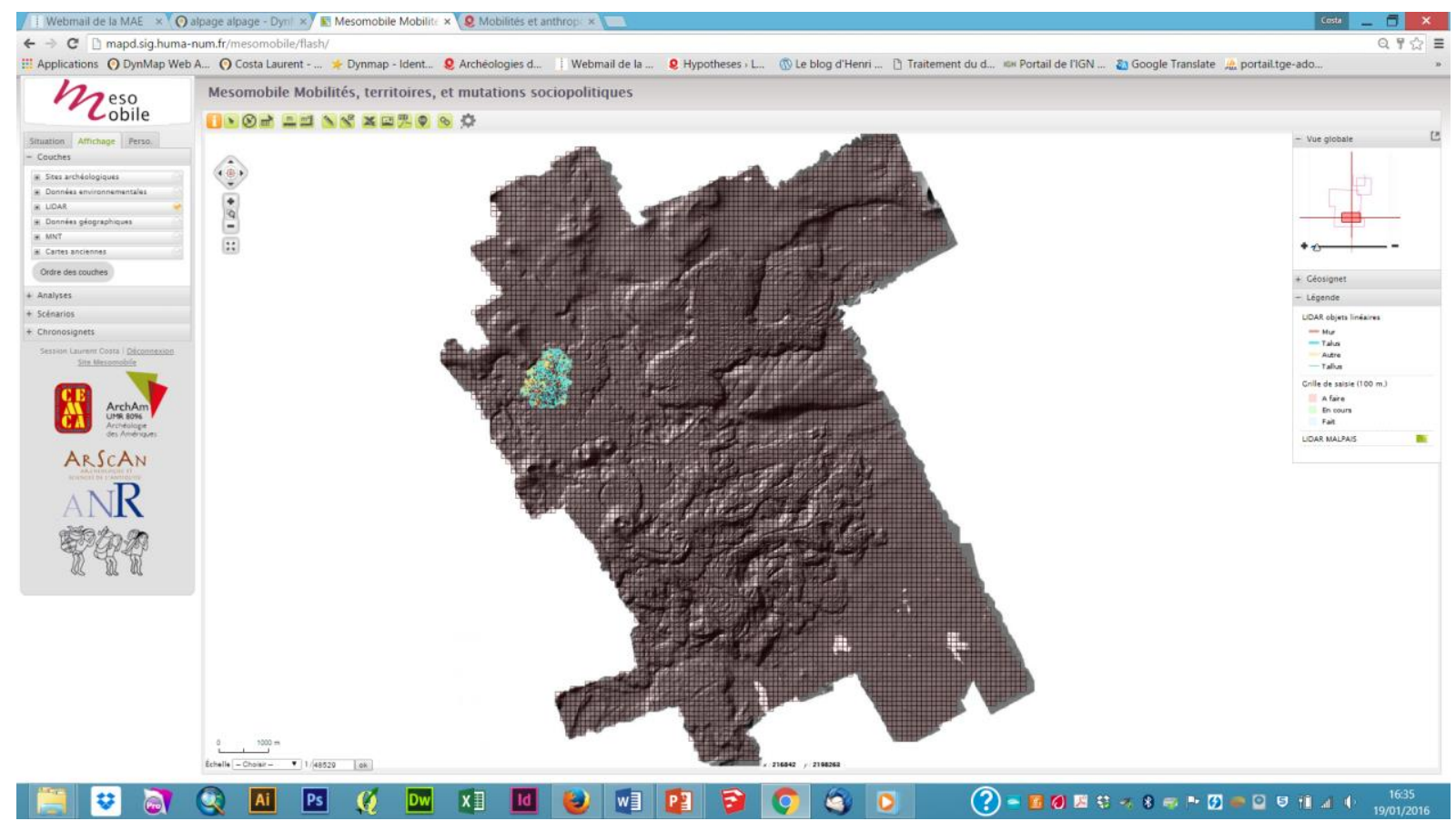

Figure 5. L'interface Mesomobile dans la plateforme Reagit du programme ArchéoFab

Chaque participant a donc reçu un accès personnel à l'application. Après une présentation en présentiel détaillée de la typologie architecturale et urbanistique connue pour El Infiernillo et en se basant sur les couches de données LiDAR proposées à savoir l'ombrage et les pentes, les participants de l'initiative ont saisi les données selon le rythme de chacun. Ils ont dessiné, sous forme vectorielle, les vestiges architecturaux composants le site. L'interprétation a été sectorisée par unité de $100 \mathrm{~m}$ sur $100 \mathrm{~m}$. L'entité saisie, uniquement sous forme linéaire, pouvait être, une fois dessinée, classée dans trois couches d'information : "mur", "talus" (les tracés linéaires: talus, terrasses, segments circulatoires) ou "autre". La première catégorie se présente sous la forme d'un élément linéaire plan et étroit (largeur généralement d'un pixel), correspondant au sommet du mur, flanqué de deux zones 
inclinées assez étroites ( 2 à 4 pixels de large) correspondant au talus d'éboulis du même mur situé de part et d'autre de celui-ci. Ce type d'élément délimite souvent un espace fermé quadrangulaire ou circulaire qui correspond à l'intérieur d'un bâtiment cellulaire. La seconde catégorie («talus») correspond à des tracés linéaires rectilignes ou subrectilignes défini par la jonction entre un espace plan plus ou moins vaste et de forme variable, supposément nivelé artificiellement, et un plan sub vertical ou incliné identifié comme la contention du nivellement. Ces éléments peuvent ainsi délimiter des terrasses ou des espaces de circulation (tracés généralement ouverts) ou encore de véritables plateformes (tracés fermés). La dernière catégorie ("autres») désigne l'ensemble des anomalies topographiques qui semblent artificielles mais dont la configuration ne correspond à aucune des précédentes.

\section{Le cas d'étude : l'agglomération archéologique d'El Infiernillo}

C'est donc sur le site archéologique d'El Infiernillo, moins étudié que ses voisins en raison de son importante surface, que se sont concentrés nos tests méthodologiques. Ce grand établissement, occupé entre 1250 et 1450 après J.-C. est localisé dans le secteur ouest du Malpaís de Zacapu, sur une coulée volcanique aujourd'hui relativement boisée. Le sol du site est contraignant pour la prospection pédestre, car très irrégulier et la roche de Malpaís affleurant partout, de manière irrégulière, sans toutefois nécessiter un aménagement aussi magistral que le site voisin de Malpaís Prieto. Ce cas était particulièrement adapté à l'exercice: il s'agissait d'un site archéologique connu, pour lequel nous disposions d'informations générales, sur les vestiges et l'environnement. La typologie architecturale était en grande partie maîtrisée et un inventaire général des structures avait été réalisé, permettant une carte de distribution globale des structures d'habitat et des temples-pyramides, balisant les espaces civico-cérémoniels. Cependant, nous ne disposions pas d'un relevé complet du site, permettant une analyse fine de l'organisation urbaine de cet établissement, le plus important du Malpaís de Zacapu en terme de surface (140 hectares estimés en 1994) et d'effectif (1154 édifices «cellulaires » et 22 temples-pyramides estimés). Le relevé pédestre par GPS et l'enregistrement architectural systématique réalisé en 2010, avait permis l'obtention du plan de huit hectare, sur la base d'une couverture moyenne de 1 ha/jour (moyenne identique à Malpaís Prieto). Sur cette base, le relevé complet du site selon cette méthodologie, pourtant considérée comme la plus économique et rapide, était estimé à 142 jours supplémentaires, soit presque cinq mois de travail.

\section{Bilan de la vectorisation collaborative en ligne et vérifications en laboratoire}

A l'issue de la saisie collaborative, répartie sur 47 jours et menée par 20 intervenants différents (Tableau 2), 3311 entités ont été créées (1 136 «talus », 1633 «murs » et 542 « autres ») sur la seule base, bien sûr, de la donnée télé-détectée, et une connaissance générale de la typologie architecturale du site.

\begin{tabular}{|c|c|c|c|}
\hline \multicolumn{2}{|c|}{$\mathbf{N}$ d'entités saisies/jour } & Moyenne & 44 \\
\hline moyenne & 72 & Médiane & 28 \\
\hline médiane & 47 & Max & 409 \\
\hline $\max$ & 424 & Min & 2 \\
\hline $\min$ & 2 & & 2 \\
\hline
\end{tabular}

Tableau 2. Synthèse statistiques de la saisie collaborative 


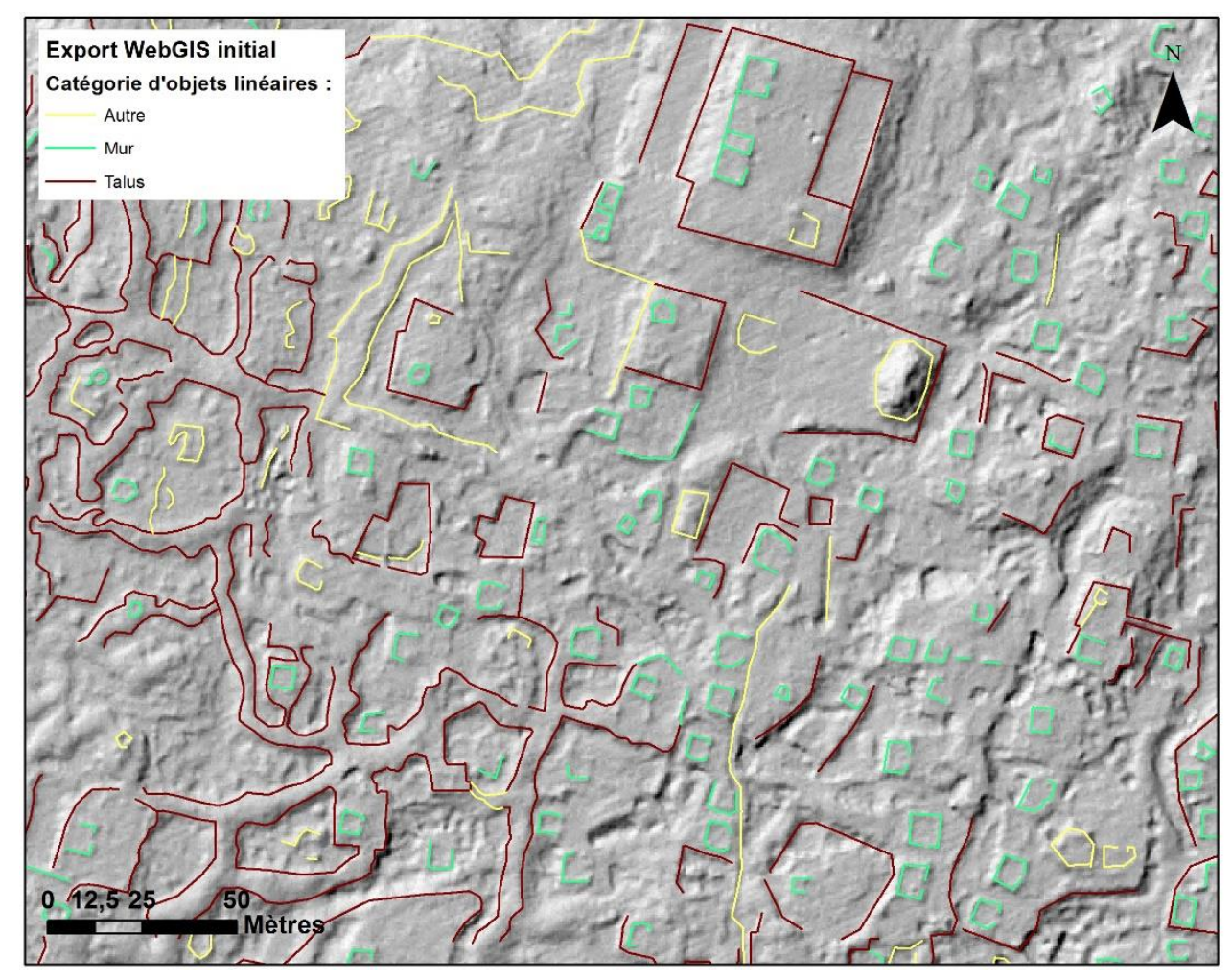

Figure 6. Détail de la vectorisation collaborative des vestiges du secteur du temple-pyramide Y-2 à El Infiernillo.

Nous avons ensuite procédé à deux étapes de vérification successives : la première réalisée par Madeleine Tisserand, l'une des étudiantes ayant participé à la saisie en ligne, toujours sans connaissance directe du terrain ou du contexte archéologique et la seconde vérification, par Marion Forest, responsable de la cartographie des agglomérations du Malpaís entre 2008 et 2010. Il s'agissait de vérifier, entité par entité, les modalités de saisie et l'interprétation des éléments. Un travail indispensable de lissage, de repositionnement de plusieurs entités dans l'une ou l'autre des couches préétablies, de compléments et de suppressions a été effectué. Ces vérifications se sont portés sur le principal type architectural composant le site : les édifices «cellulaires », généralement enregistrés dans la catégorie «murs» et définis par un mur de contour continu ou discontinu (porte) quadrangulaire ou circulaire, délimitant une pièce simple (les possibles subdivisions observées in situ sont invisibles sur le LiDAR).

Ces édifices qui se présentent aujourd'hui comme de petits enclos composant la très large partie du bâti des agglomérations du Malpaís (plus de 68\% à Malpaís Prieto) et sont principalement des structures à vocation domestiques. Ils forment, par leur effectif et leur distribution, la population statistique la plus parlante de ces unités urbaines.

A l'issue de la première phase de cette vérification (deux semaines), qui consistait à isoler ces objets du reste de la saisie collective et procéder à une vérification fine de l'ensemble de leur saisie, l'inventaire s'élevait à 1544 entités, soit 89 édifices de moins que la saisie collective, mais toujours 390 individus de plus que l'inventaire assez systématique réalisé en 1994 ( $\mathrm{n}=1154)$. Bien qu'une cinquantaine de structures aient été repérées hors des limites prospectées en 1994, la différence d'inventaire dépassait les 300 édifices. 


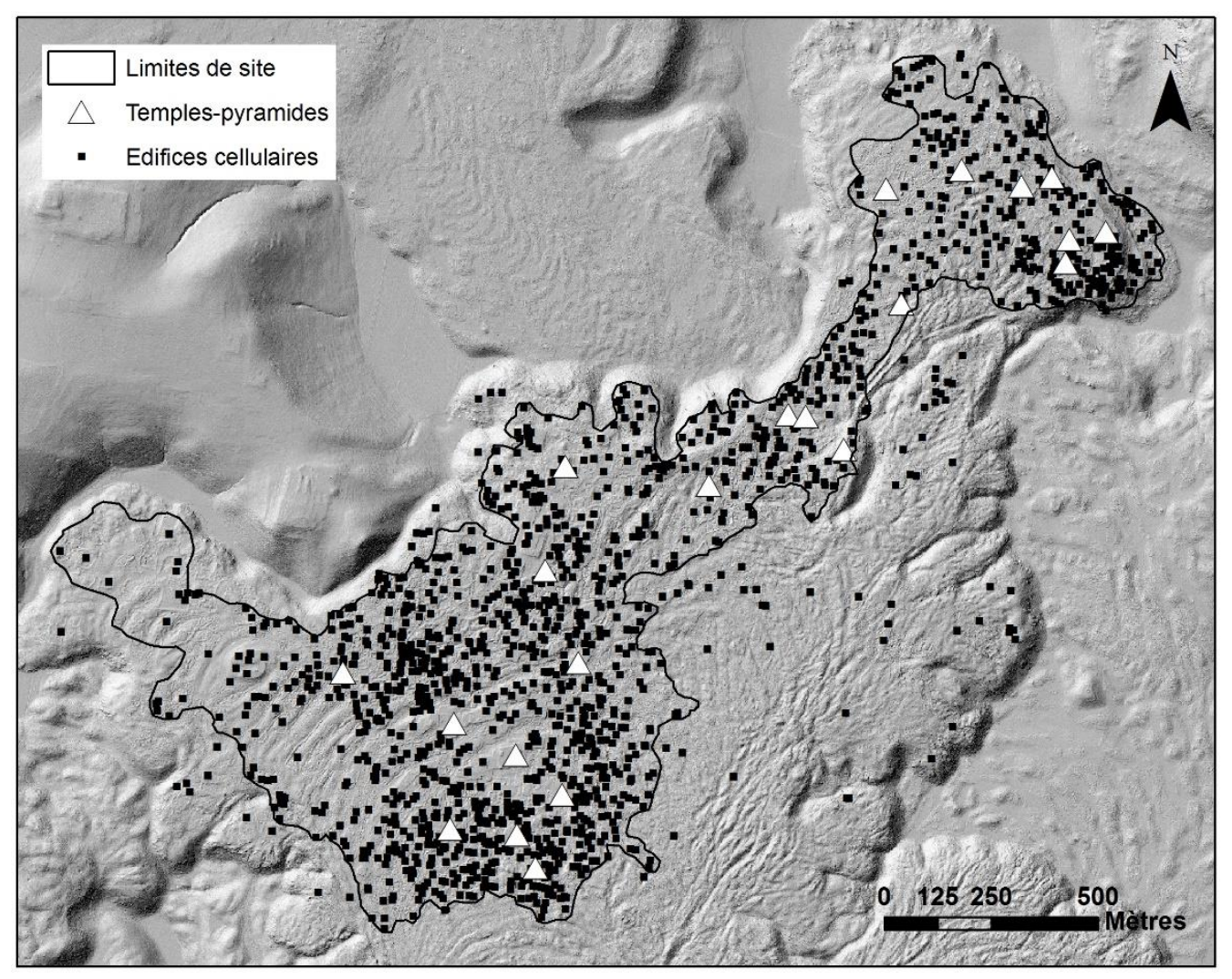

Figure 7. Distribution des édifices cellulaires et des temples-pyramides à El Infiernillo après la 1ère étape de vérification.

A l'issue de la deuxième vérification (une journée de travail), cette fois-ci menée par une spécialiste de ce terrain, l'inventaire des édifices cellulaires s'élève à 1034 individus parfaitement identifiés, auxquels s'ajoutent 188 individus dont l'état de conservation/de lecture est moins bon, soit un total de 1222 édifices cellulaires. Au total, 328 entités ont été supprimées : il s'agissait de doublons (deux enregistrements pour le même édifice), des erreurs d'interprétation (autre type de vestiges), ou des structures si mal définies qu'elles ne pouvaient être validées. L'inventaire final vérifié semble cohérent avec l'enregistrement global réalisé en 1994 qui s'impose ici comme un très bon référent de « rétroanalyse ». La différence s'explique par deux principaux aspects. D'une part, malgré les vérifications, une marge d'erreur reste présente dans la télédétection des vestiges. D'autre part, la périphérie de l'agglomération est plus facilement explorable par observation du modèle de terrain qu'en 1994 sur le terrain : la prise en compte des entités présentes « hors les murs » est possible. 


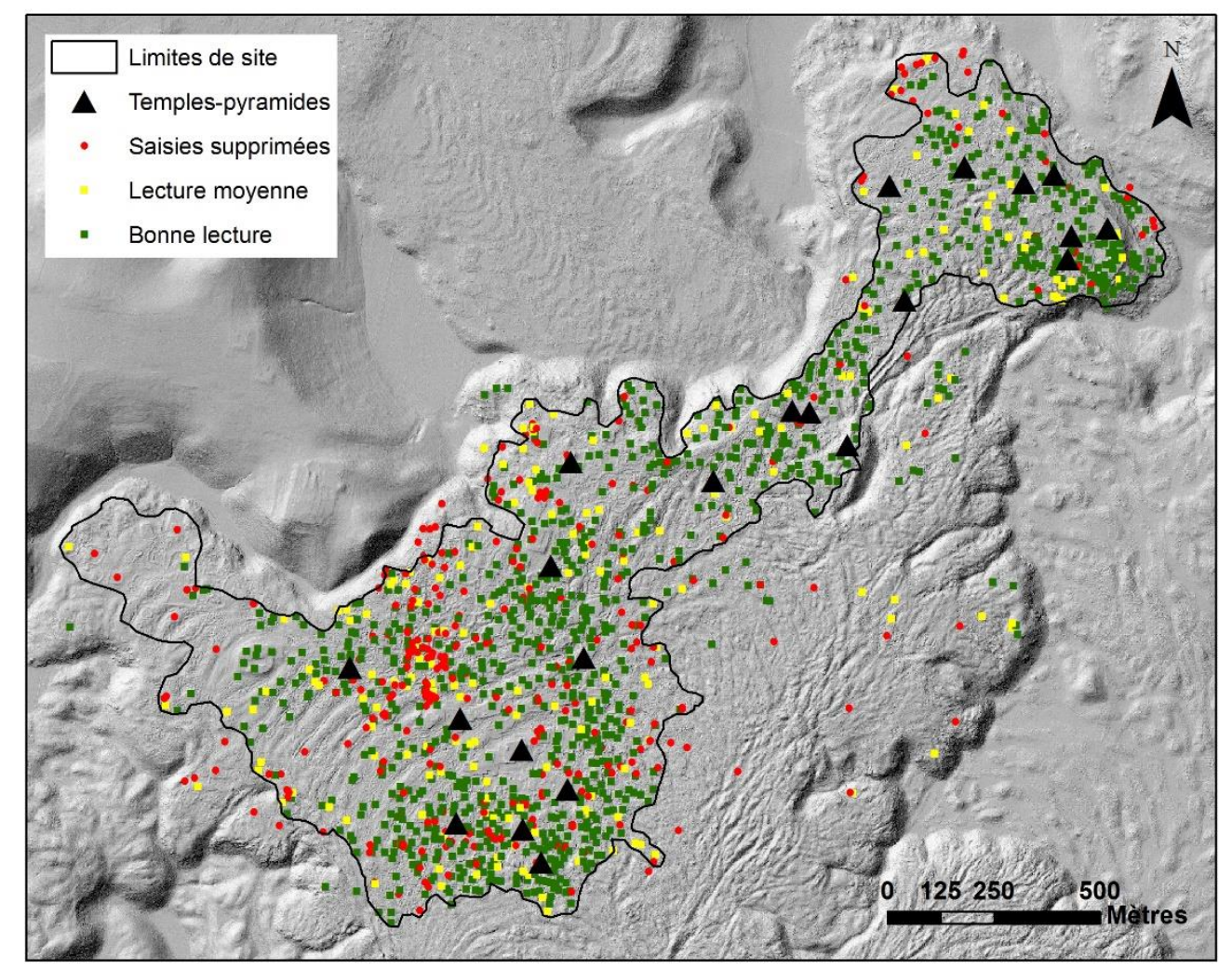

Figure 8. Distribution des édifices cellulaires et des temples-pyramides à El Infiernillo après la deuxième étape de vérification de la saisie collaborative.

\section{Rétro-validation : les données de terrain}

Le troisième test correspond à une courte campagne de terrain (15 jours) menée par Clara Zimmer, Marion Forest et Grégory Pereira. L'objectif de cette opération était de cartographier en détail un secteur d'El Infiernillo (possédant une bonne représentativité architecturale) et en particulier d'identifier et interpréter les formes linéaires, très nombreuses sur le site et mal comprises (Zimmer 2016). Environ 5 ha du site, correspondant au secteur du temple-pyramide Y22 ont donc été étudiés en détails, permettant de confronter les interprétations «à l'aveugle » faites à partir des données LiDAR à la réalité des vestiges archéologiques.

\begin{tabular}{|c|c|c|c|c|c|c|c|}
\hline & $\begin{array}{c}\text { Formes } \\
\text { linéaires }\end{array}$ & $\begin{array}{c}\text { Terrasses } \\
\text { et talus }\end{array}$ & $\begin{array}{c}\text { Edifices } \\
\text { cellulaires }\end{array}$ & $\begin{array}{c}\text { Dépressions } \\
\text { circulaires }\end{array}$ & $\begin{array}{c}\text { Structure } \\
\text { atypique }\end{array}$ & $\begin{array}{c}\text { Soubassement } \\
\text { pyramidal }\end{array}$ & Total \\
\hline $\begin{array}{c}\text { Interprétation } \\
\text { LiDAR « à } \\
\text { l'aveugle » }\end{array}$ & 56 & 80 & 23 & 13 & - & 1 & 184 \\
\hline $\begin{array}{c}\text { Prospection } \\
\text { pédestre }\end{array}$ & 48 & 116 & 25 & 21 & 14 & 1 & 225 \\
\hline
\end{tabular}

Tableau 3. Tableau de comparaison entre les entités découvertes à travers la donnée LiDAR et les prospections du secteur du temple-pyramide Y22 (d'après Zimmer 2016 : 72).

Le premier constat est sans doute la bonne identification des édifices cellulaires (deux individus supplémentaires, de taille réduite, ont été ajoutés à l'inventaire général) et des temples-pyramides. La lecture des types plus hétérogènes tels que les monticules, les dépressions naturelles intégrées dans l'urbanisme, et le linéaire (terrassement, segments circulatoires, espaces aménagés partiellement), quant à elle, s'avère plus complexe : la variété des formes et des formats, ainsi que l'irrégularité du 
terrain naturel rendent l'interprétation de l'image LiDAR impossible. Force est de constater, que même l'analyse du paysage et du bâti sur le terrain, est-elle aussi inégale et peu claire.

\section{Conclusions}

Rappelons en guise de conclusion que l'un des apports essentiels du LiDAR est l'appréhension globale des sites qu'il permet. En revanche, il ne faut pas oublier les contraintes de ces nouveaux supports qui nécessitent la mise en place de stratégies de recherche adaptées. Notons que dans cette optique, la question des interfaces n'est pas anecdotique et qu'elle détermine aussi les possibilités d'analyses potentielles. L'option proposée ici est celle d'une approche via une interface cartographique permettant de la saisie collaborative. Cette interface nous a permis de couvrir rapidement des surfaces importantes et de proposer une couche de données vectorielle manipulable et dont la qualité globale était satisfaisante au regard des données terrain connues et des vérifications que nous avons pu réaliser.

Mais la possibilité d'interpréter en «aveugle» une donnée, ici du LiDAR, pose cependant la question des modalités de structuration et d'interprétation de ces formes brutes... à priori, une interprétation d'une donnée issue d'un capteur (LiDAR ou autre) qu'elle soit issue d'un traitement collaboratif tel qu'ici ou même lorsqu'il s'agit d'une approche plus algorithmique (issue du calcul) nécessite un questionnement sur la valeur de cette extraction. De fait, l'interprétation " hors terrain » de ces données nous fournit, non pas un plan archéologique, mais des informations morphologiques (lignes, points et surfaces) éventuellement pré-caractérisées (murs, terrasses, autres). Cet ensemble ne peut pas être considéré autrement que comme une pré-interprétation d'autant qu'il ne s'agit que d'un échantillonnage partiel et en quelque sorte partial, le LiDAR comme tout capteur, ne donnant à voir qu'une partie de la réalité archéologique (cette réalité est largement dépendante de la définition du modèle et du type de capteur utilisé...ici des pixels de $50 \mathrm{~cm}$ ). L'étape suivante est donc forcément celle de la caractérisation (fonction / datation) de ces indices morphologiques et nécessite un retour terrain comme l'illustre notre exemple. La différence étant ici que cette visite de terrain est préparée par l'interprétation.

Par ailleurs le LiDAR nous offre un continuum d'information qui n'est pas sans questionner notre démarche. Loin d'être un détail, la question de la masse d'information et surtout de sa continuité spatiale implique de réfléchir au mode de segmentation : où s'arrête la structure, où s'arrête le site ? Ici, il est à nouveau important de comprendre que seule l'interprétation archéologique permet de donner sens à des données brute, issues des capteurs et qui ne livrent d'autre choses que des mesures ou des dérivées de ces mesures... Le rôle de l'expertise archéologique s'en trouve alors renforcée pour que d'informations sortent des connaissances...

Au final, l'expérience présentée ici apporte de multiple indications. Archéologiquement d'abord, elle nous a permis de percevoir les structures globales du site malgré les limites liées à la collecte de la donnée et à son interprétation. En découle la confirmation rapide de schémas d'établissement suspectés sur la base d'études pilotes ou de travaux comparatifs. Nous avons aussi observé l'importance et la richesse d'une approche hybride et complémentaire menée sur le Malpaís de Zacapu, reposant à la fois sur l'analyse algorithmique et sur l'interprétation des experts. Enfin, cette expérience a démontré son fort potentiel pédagogique : une formation à l'exercice d'interprétation archéologique des données LIDAR par la pratique collective. 


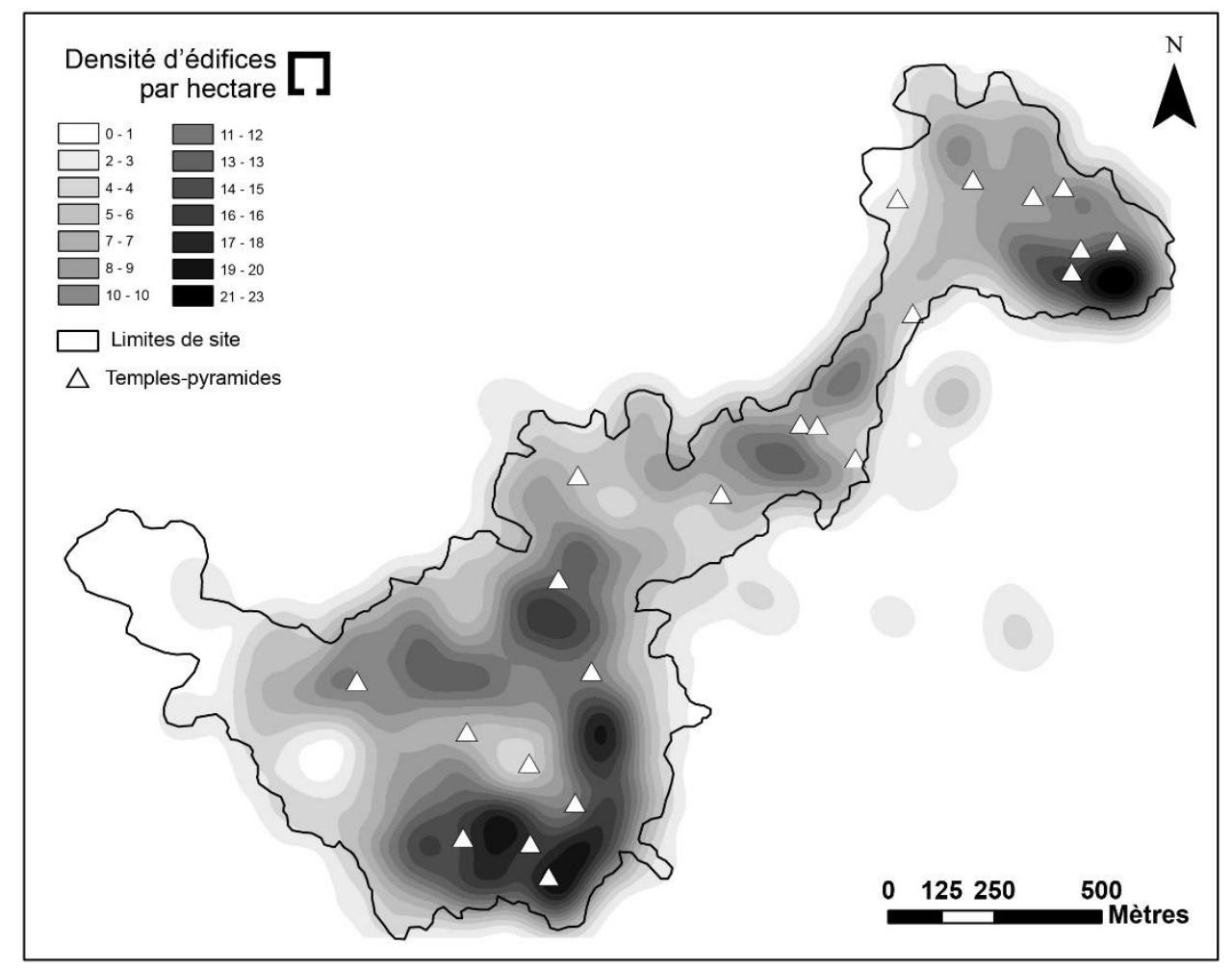

Figure 9. Carte de densité des édifices « cellulaires » à El Infiernillo réalisée après traitement des données LiDAR.

\section{Références}

Caso, Alfonso

1930 Informe preliminar de las exploraciones efectuadas en Michoacán. Anales del Museo Nacional de Mexico 4a época 6 (2):446-452.

Costa, Laurent \& Desachy, Bruno

2018 ArcheoFab - Archéologies du Bassin Parisien. Culture et recherche, $\mathrm{n}^{\mathrm{o}}$ 137, p. 31-32.

Fisher, Christopher \& Stephen Leisz

2013 New Perspectives on Purépecha Urbanism Through the Use of Lidar at the Site of Angamuco, Mexico. In Space Archaeology: Mapping Ancient Landscapes with Air and Spaceborne Imagery, D. M. Harrower (Ed.), pp. 191-201. Springer, New York.

Forest, Marion

2008 Identification et utilisation des espaces du site du Malpaís Prieto, Michoacán, Mexique. Mémoire de Master 2, Université de Paris 1 Panthéon-Sorbonne, Paris.

2014 Approches spatio-archéologiques de la structure sociale des sites urbains du Malpaís de Zacapu. Thèse de Doctorat, Université de Paris 1 - Panthéon/Sorbonne, Paris.

Freddolino, Marie K.

1973 An investigation into the pre-Tarascan cultures of Zacapu, Michoacán, Mexico. PhD dissertation, Yale University, New-Haven.

Golden, Charles, Timothy Murtha, Bruce Cook, Derek S. Shaffer, Whittaker Schroder, Elijah J. Hermitt, Omar Alcover Firpi and Andrew K. Scherer

2016 Reanalyzing environmental lidar data for archaeology: Mesoamerican applications and implications. Journal of Archaeological Science 9:293-308. 
Lumholtz, Carl

1986 [1904] El México desconocido. (facsimile edition by Charles Scribner's Son, New York, 1904). 2 vols. Instituto Nacional Indigenista, Mexico.

Michelet, Dominique

1998 Topografía y prospección sistemática de los grandes asentamientos del Malpaís de Zacapu: claves para un acercamiento a las realidades sociopolíti- cas. In Génesis, culturas y espacios en Michoacán, edited by V. Darras, pp. 4759. CEMCA, México.

Michelet, Dominique, Alain Ichon et Gérald Migeon

1988 Residencias, barrios y sitios posclásicos en el Malpais de Zacapu. In Primera reunión sobre les sociedades prehispánicas en el Centro-Occidente de México. Cuaderno de trabajo 1, pp. 177-191. INAH, Querétaro.

Michelet, Dominique

1992 El Centro-Norte de Michoacán: características generales de su estudio regional. In El Proyecto Michoacán 1983-1987. Medio ambiente e introducción a los trabajos arqueológicos, pp. 9-52. CEMCA, Mexico.

Migeon, Gérald

1990 Archéologie en pays tarasque. Structure de l'habitat et ethnopréhistoire des habitations tarasques de la région de Zacapu (Michoacan, Mexique) au Postclassique Récent. Tesis doctoral. UFR d'art et d'archéologie, Université de Paris 1, México.

Pereira, Grégory \& Marion Forest

2011 Informe sobre los trabajos de campo realizados en el sitio El Malpaís Prieto, Michoacán, México: temporada 3 (Febrero-Mayo del 2010) UMR 8096, CNRS, 125 p.

Pereira, Grégory, Marion Forest, Dominique Michelet \& Elsa Jadot

2012 Proyecto Uacúsecha. Informe sobre los trabajos de campo realizados en el sitio El Malpaís Prieto y otros asentamientos de la región de Zacapu, Michoacán : temporada 4 (2011-2012). UMR 8096, CNRS, 103 p., 69 figs.

Pereira, Grégory, Dominique Michelet, Antoine Dorison, Brigitte Faugère, Osiris Quezada, Karine Lefebvre, Marion Forest, Isabel Medina, Isaac Barrientos, Hemmamuthé Goudiaby, Elsa Jadot, Aurélie Manin, Luis Barba, Jorge Blancas, Agustín Ortiz \& Céline Gillot

2016 Proyecto Uacúsecha. Informe sobre los trabajos de campo realizados en el sitio El Malpaís Prieto y otros asentamientos de la región de Zacapu, Michoacán : temporada 8 (2015-2016). UMR 8096, CNRS, 262 p., 215 figs.

Zimmer, Clara

2016 Réseau Viaire ou parcellaire ? L'apport du LIDAR à l'analyse des structures linéaires du site d'El Infernillo, Michoacán, Mexique (1250-1450 apr. J.C.). Mémoire de Master 2, Université de Paris 1, Panthéon-Sorbonne.

\section{Remerciements}

Le présent travail s'inscrit dans le cadre du programme Mésomobile (ANR-14-CE31-0016 porté par Véronique Darras) et du projet archéologique Uacúsecha (financé par le Ministère de l'Europe et des Affaires Etrangères et dirigé par Grégory Pereira). Il a bénéficié du soutien du Labex Dynamite.

Christophe Petit, Professeur d'archéologie environnementale à l'Université Paris 1 Panthéon Sorbonne a permis la réalisation de la formation WebGIS et la saisie collaborative dans le cadre de son séminaire de Master 2 «Pratiques de l'archéologie environnementale : laboratoire ». 
Ont participé à la saisie: Nicolas Bermeo, Hugo Cador, Marion Connan, Ana Cremont, Sarah Davidoux, Claudia Dias, Djama Amari, Clément Membrives, Angélique Montes, Philippe Rajat, Madeleine Tisserand, Bastien Torres, Christophe Tuffery et Clara Zimmer. Cette dernière a activement participé à la campagne de vérification sur le terrain en avril 2015 et rédigé, sur cette base, son mémoire de Master 2. 